\title{
Home appliances management system using Controller Area Network (CAN)
}

\begin{abstract}
Smart homes can be a potential application which provides unobtrusive support for the normal users, elderly or disabled that wants to promote independent living. Generally, smart home consists of various sensors, controllers and home network that are placed around the house. This paper presents a simple home networking system by using Controller Area Network (CAN) as the main communication protocol to manage multiple home appliances. The proposed management system consists of three main components: central controller subsystem (CCS), CAN module and relay module. CCS is a simple server where a simple graphical user interface (GUI) is built with multiple command buttons to obtain the user inputs. The CAN module is distinguished as either master CAN node or slave CAN node. The master CAN node is a CAN module that is attached to CCS. In contrast, the CAN module that attached to the home appliance is known as slave CAN node. The user input signal that contains a CAN data frame broadcasts from master CAN node to all slave CAN nodes via CAN bus. Each data frame consists of an identifier that is compared by each slave CAN node. If the identifier is not matched, the signal will be discarded by the slave CAN node. Otherwise, the slave CAN node will decode the data frame and execute the given command. The relay module of this slave CAN node will either switch 'ON' or 'OFF' the corresponding home appliances. The proposed management system using CAN has the advantages of being simple in its design which contributes to the overall low cost. Moreover, the CAN network system is immune from the electrical interferences. Finally, the experimental results have shown that the proposed system is operating accurately and efficiently.
\end{abstract}

Keyword: CAN; Home appliances; Identifier 